\title{
Study on integro-differential equation with generalized $p$-Laplacian operator
}

\author{
Li Wei ${ }^{1}$, Ravi P Agarwal ${ }^{2,3^{*}}$ and Patricia JY Wong ${ }^{4}$
}

\section{"Correspondence:}

agarwal@tamuk.edu

${ }^{2}$ Department of Mathematics, Texas

A\&M University - Kingsville,

Kingsville, TX 78363, USA

${ }^{3}$ Department of Mathematics,

Faculty of Science, King Abdulaziz

University, Jeddah, 21589, Saudi

Arabia

Full list of author information is

available at the end of the article

\begin{abstract}
We tackle the existence and uniqueness of the solution for a kind of integro-differential equations involving the generalized $p$-Laplacian operator with mixed boundary conditions. This is achieved by using some results on the ranges for maximal monotone operators and pseudo-monotone operators. The method used in this paper extends and complements some previous work.
\end{abstract}

MSC: $47 \mathrm{H} 05 ; 47 \mathrm{HO}$

Keywords: maximal monotone operator; pseudo-monotone operator; generalized p-Laplacian operator; integro-differential equation; mixed boundary conditions

\section{Introduction}

Nonlinear boundary value problems (BVPs) involving the $p$-Laplacian operator $-\Delta_{p}$ arise from a variety of physical phenomena such as non-Newtonian fluids, reaction-diffusion problems, petroleum extraction, flow through porous media, etc. Thus, the study of such problems and their generalizations have attracted numerous attention in recent years. Some of the BVPs studied in the literature include the following:

$$
\left\{\begin{array}{l}
-\Delta_{p} u+g(x, u(x))=f(x), \quad \text { a.e. in } \Omega \\
-\frac{\partial u}{\partial n}=0, \quad \text { a.e. on } \Gamma
\end{array}\right.
$$

whose existence results in $L^{p}(\Omega)$ (for various ranges of $p$ ) can be found in [1-4]; a related BVP

$$
\left\{\begin{array}{l}
-\Delta_{p} u+g(x, u(x))=f(x), \quad \text { a.e. in } \Omega, \\
-\left\langle\vartheta,|\nabla u|^{p-2} \nabla u\right\rangle \in \beta_{x}(u(x)), \quad \text { a.e. on } \Gamma
\end{array}\right.
$$

was tackled in [5-7] and later generalized to one that contains a perturbation term $|u|^{p-2} u$ $[8,9]$

$$
\left\{\begin{array}{l}
-\Delta_{p} u+|u|^{p-2} u+g(x, u(x))=f(x), \quad \text { a.e. in } \Omega, \\
-\left\langle\vartheta,|\nabla u|^{p-2} \nabla u\right\rangle \in \beta_{x}(u(x)), \quad \text { a.e. on } \Gamma .
\end{array}\right.
$$

\section{照 Springer}

(O) 2012 Wei et al.; licensee Springer. This is an Open Access article distributed under the terms of the Creative Commons Attribution License (http://creativecommons.org/licenses/by/2.0), which permits unrestricted use, distribution, and reproduction in any medium, provided the original work is properly cited. 
Motivated by Tolksdorf's work [10] where the following Dirichlet BVP has been discussed:

$$
\left\{\begin{array}{l}
-\operatorname{div}\left[\left(C(x)+|\nabla u|^{2}\right)^{\frac{p-2}{2}} \nabla u\right]=f(x), \quad \text { a.e. } \operatorname{in} K(1, S), \\
u=g, \quad \text { a.e. } \operatorname{in} \Sigma(1, S)
\end{array}\right.
$$

several generalizations have been investigated. These include [11-14]

$$
\begin{aligned}
& \left\{\begin{array}{l}
-\operatorname{div}\left[\left(C(x)+|\nabla u|^{2}\right)^{\frac{p-2}{2}} \nabla u\right]+|u|^{p-2} u+g(x, u(x))=f(x), \quad \text { a.e. in } \Omega, \\
-\left\langle\vartheta,\left(C(x)+|\nabla u|^{2}\right)^{\frac{p-2}{2}} \nabla u\right\rangle=0, \quad \text { a.e. on } \Gamma,
\end{array}\right. \\
& \left\{\begin{array}{l}
-\operatorname{div}\left[\left(C(x)+|\nabla u|^{2}\right)^{\frac{p-2}{2}} \nabla u\right]+|u|^{p-2} u+g(x, u(x))=f(x), \quad \text { a.e. in } \Omega, \\
-\left\langle\vartheta,\left(C(x)+|\nabla u|^{2}\right)^{\frac{p-2}{2}} \nabla u\right\rangle \in \beta_{x}(u(x)), \quad \text { a.e. on } \Gamma
\end{array}\right.
\end{aligned}
$$

and

$$
\left\{\begin{array}{l}
-\operatorname{div}\left[\left(C(x)+|\nabla u|^{2}\right)^{\frac{p-2}{2}} \nabla u\right]+\varepsilon|u|^{q-2} u+g(x, u(x))=f(x), \quad \text { a.e. in } \Omega, \\
-\left\langle\vartheta,\left(C(x)+|\nabla u|^{2}\right)^{\frac{p-2}{2}} \nabla u\right\rangle \in \beta_{x}(u(x)), \quad \text { a.e. on } \Gamma
\end{array}\right.
$$

where $0 \leq C(x) \in L^{p}(\Omega), \varepsilon$ is a nonnegative constant and $\vartheta$ denotes the exterior normal derivative of $\Gamma$.

Inspired by all this research, recently we have studied the following nonlinear parabolic equation with mixed boundary conditions [15]:

$$
\left\{\begin{array}{l}
\frac{\partial u}{\partial t}-\operatorname{div}\left[\left(C(x, t)+|\nabla u|^{2}\right)^{\frac{p-2}{2}} \nabla u\right]+\varepsilon|u|^{p-2} u=f(x, t), \quad(x, t) \in \Omega \times(0, T), \\
-\left\langle\vartheta,\left(C(x, t)+|\nabla u|^{2}\right)^{\frac{p-2}{2}} \nabla u\right\rangle \in \beta(u)-h(x, t), \quad(x, t) \in \Gamma \times(0, T), \\
u(x, 0)=u(x, T), \quad \text { a.e. } x \in \Omega .
\end{array}\right.
$$

We tackle the existence of solutions for (1.8) via the study of existence of solutions for two BVPs: (i) the elliptic equation with Dirichlet boundary conditions

$$
\left\{\begin{array}{l}
-\operatorname{div}\left[\left(C(x)+|\nabla u|^{2}\right)^{\frac{p-2}{2}} \nabla u\right]+\varepsilon|u|^{q-2} u=f(x), \quad \text { a.e. in } \Omega, \\
\gamma u=w, \quad \text { a.e. on } \Gamma
\end{array}\right.
$$

and (ii) the elliptic equation with Neumann boundary conditions

$$
\left\{\begin{array}{l}
-\operatorname{div}\left[\left(C(x)+|\nabla u|^{2}\right)^{\frac{p-2}{2}} \nabla u\right]+\varepsilon|u|^{q-2} u=f(x), \quad \text { a.e. in } \Omega, \\
-\left\langle\vartheta,\left(C(x)+|\nabla u|^{2}\right)^{\frac{p-2}{2}} \nabla u\right\rangle \in \beta(u)-h(x), \quad \text { a.e. in } \Gamma .
\end{array}\right.
$$

By setting up the relations between the auxiliary equations (1.9) and (1.10) and by employing some results on ranges for maximal monotone operators, we showed that (1.8) has a unique solution in $L^{p}\left(0, T ; W^{1, p}(\Omega)\right)$, where $2 \leq p<+\infty, 1 \leq q<+\infty$ if $p \geq N$, and $1 \leq q \leq \frac{2 N-p}{N-p}$ if $p<N$.

In this paper, we shall employ the technique used in (1.8), viz. using the results on ranges for nonlinear operators, to study the existence and uniqueness of the solution to a nonlinear integro-differential equation with the generalized $p$-Laplacian operator. We note that 
most of the existing methods in the literature used to investigate such problems are based on the finite element method, hence our technique is new in tackling integro-differential equations. We shall consider the following nonlinear integro-differential problem with mixed boundary conditions:

$$
\left\{\begin{array}{l}
\frac{\partial u}{\partial t}-\operatorname{div}\left[\left(C(x, t)+|\nabla u|^{2}\right)^{\frac{p-2}{2}} \nabla u\right]+\varepsilon|u|^{q-2} u+a \frac{\partial}{\partial t} \int_{\Omega} u d x \\
\quad=f(x, t), \quad(x, t) \in \Omega \times(0, T), \\
-\left\langle\vartheta,\left(C(x, t)+|\nabla u|^{2}\right)^{\frac{p-2}{2}} \nabla u\right\rangle \in \beta_{x}(u), \quad(x, t) \in \Gamma \times(0, T), \\
u(x, 0)=u(x, T), \quad x \in \Omega .
\end{array}\right.
$$

Our discussion is based on some results on the ranges for maximal monotone operators and pseudo-monotone operators in [16-18]. Some new methods of constructing appropriate mappings to achieve our goal are employed. Moreover, we weaken the restrictions on $p$ and $q$. The paper is outlined as follows. In Section 2 we shall state the definitions and results needed, and in Section 3 we shall establish the existence and uniqueness of the solution to (1.11).

\section{Preliminaries}

Let $X$ be a real Banach space with a strictly convex dual space $X^{*}$. We use $(\cdot, \cdot)$ to denote the generalized duality pairing between $X$ and $X^{*}$. For a subset $C$ of $X$, we use Int $C$ to denote the interior of $C$. We also use ' $\rightarrow$ ' and ' $w$-lim' to denote strong and weak convergences, respectively.

Let $X$ and $Y$ be Banach spaces. We use $X \hookrightarrow Y$ to denote that $X$ is embedded continuously in $Y$.

The function $\Phi$ is called a proper convex function on $X$ [17] if $\Phi$ is defined from $X$ to $(-\infty,+\infty], \Phi$ is not identically $+\infty$ such that $\Phi((1-\lambda) x+\lambda y) \leq(1-\lambda) \Phi(x)+\lambda \Phi(y)$, whenever $x, y \in X$ and $0 \leq \lambda \leq 1$.

The function $\Phi: X \rightarrow(-\infty,+\infty]$ is said to be lower-semicontinuous on $X$ [17] if $\liminf _{y \rightarrow x} \Phi(y) \geq \Phi(x)$ for any $x \in X$.

Given a proper convex function $\Phi$ on $X$ and a point $x \in X$, we denote by $\partial \Phi(x)$ the set of all $x^{*} \in X^{*}$ such that $\Phi(x) \leq \Phi(y)+\left(x-y, x^{*}\right)$ for every $y \in X$. Such elements $x^{*}$ are called subgradients of $\Phi$ at $x$, and $\partial \Phi(x)$ is called the subdifferential of $\Phi$ at $x$ [17].

A mapping $T: D(T)=X \rightarrow X^{\prime \prime}$ is said to be demi-continuous on $X$ if $w$ - $\lim _{n \rightarrow \infty} T x_{n}=T x$ for any sequence $\left\{x_{n}\right\}$ strongly convergent to $x$ in $X$. A mapping $T: D(T)=X \rightarrow X^{*}$ is said to be hemi-continuous on $X$ if $w$ - $\lim _{t \rightarrow 0} T(x+t y)=T x$ for any $x, y \in X$ [17].

With each multi-valued mapping $A: X \rightarrow 2^{X}$, we associate the subset $A^{0}$ as follows [17]:

$$
A^{0} x=\{y \in A x:\|y\|=|A x|\}
$$

where $|A x|:=\inf \{\|z\|: z \in A x\}$. If $X^{*}$ is strictly convex, then $D(A)=D\left(A^{0}\right)$ and $A^{0}$ is singlevalued, which in this case is called the minimal section of $A$.

A multi-valued mapping $B: X \rightarrow 2^{X^{n}}$ is said to be monotone [18] if its graph $G(B)$ is a monotone subset of $X \times X^{*}$ in the sense that $\left(u_{1}-u_{2}, w_{1}-w_{2}\right) \geq 0$ for any $\left[u_{i}, w_{i}\right] \in G(B)$, $i=1,2$. The monotone operator $B$ is said to be maximal monotone if $G(B)$ is not properly contained in any other monotone subsets of $X \times X^{*}$. 
Definition 2.1 [18] Let $C$ be a closed convex subset of $X$, and let $A: C \rightarrow 2^{X^{*}}$ be a multivalued mapping. Then $A$ is said to be a pseudo-monotone operator provided that

(i) for each $x \in C$, the image $A x$ is a nonempty closed and convex subset of $X^{*}$;

(ii) if $\left\{x_{n}\right\}$ is a sequence in $C$ converging weakly to $x \in C$ and if $f_{n} \in A x_{n}$ is such that $\lim \sup _{n \rightarrow \infty}\left(x_{n}-x, f_{n}\right) \leq 0$, then to each element $y \in C$, there corresponds an $f(y) \in A x$ with the property that

$$
(x-y, f(y)) \leq \liminf _{n \rightarrow \infty}\left(x_{n}-x, f_{n}\right)
$$

(iii) for each finite-dimensional subspace $F$ of $X$, the operator $A$ is continuous from $C \cap F$ to $X^{*}$ in the weak topology.

Lemma 2.1 [19] Let $\Omega$ be a bounded conical domain in $\mathbb{R}^{N}$. If $m p>N$, then $W^{m, p}(\Omega) \hookrightarrow$ $C_{B}(\Omega)$; if $0<m p \leq N$ and $q=\frac{N p}{N-m p}$, then $W^{m, p}(\Omega) \hookrightarrow L^{q}(\Omega)$; if $m p=N$ and $p>1$, then for $1 \leq q<+\infty, W^{m, p}(\Omega) \hookrightarrow L^{q}(\Omega)$.

Lemma 2.2 [18] If $B: X \rightarrow 2^{X^{*}}$ is an everywhere defined, monotone, and hemi-continuous operator, then $B$ is maximal monotone. If $B: X \rightarrow 2^{X^{*}}$ is a maximal monotone operator such that $D(B)=X$, then $B$ is pseudo-monotone.

Lemma 2.3 [18] If $X$ is a Banach space and $\Phi: X \rightarrow(-\infty,+\infty$ ] is a proper convex and lower-semicontinuous function, then $\partial \Phi$ is maximal monotone from $X$ to $X^{\prime \prime}$.

Lemma 2.4 [18] If $B_{1}$ and $B_{2}$ are two maximal monotone operators in $X$ such that int $D\left(B_{1}\right) \cap D\left(B_{2}\right) \neq \emptyset$, then $B_{1}+B_{2}$ is maximal monotone.

Lemma 2.5 [20] Let $X$ and its dual $X^{\prime \prime}$ be strictly convex Banach spaces. Suppose $S: D(S) \subset$ $X \rightarrow X^{*}$ is a closed linear operator and $S^{*}$ is the conjugate operator of $S$. If $(u, S u) \geq 0$ $\forall u \in D(S)$ and $\left(v, S^{*} v\right) \geq 0 \forall v \in D\left(S^{*}\right)$, then $S$ is a maximal monotone operator possessing a dense domain.

Lemma 2.6 [18] Any hemi-continuous mapping $T: X \rightarrow X^{*}$ is demi-continuous on $\operatorname{Int} D(T)$.

Theorem 2.1 [16] Let $X$ be a real reflexive Banach space with $X^{*}$ being its dual space. Let $C$ be a nonempty closed convex subset of $X$. Assume that

(i) the mapping $A: C \rightarrow 2^{X^{*}}$ is a maximal monotone operator;

(ii) the mapping $B: C \rightarrow X^{*}$ is pseudo-monotone, bounded, and demi-continuous;

(iii) if the subset $C$ is unbounded, then the operator $B$ is $A$-coercive with respect to the fixed element $b \in X^{*}$, i.e., there exists an element $u_{0} \in C \cap D(A)$ and a number $r>0$ such that $\left(u-u_{0}, B u\right)>\left(u-u_{0}, b\right)$ for all $u \in C$ with $\|u\|>r$.

Then the equation $b \in A u+B u$ has a solution.

\section{Existence and uniqueness of the solution to (1.11)}

We begin by stating some notations and assumptions used in this paper. Throughout, we shall assume that

$$
1<q \leq p<+\infty, \quad \frac{1}{p}+\frac{1}{p^{\prime}}=1 \text { and } \quad \frac{1}{q}+\frac{1}{q^{\prime}}=1 .
$$


Let $V=L^{p}\left(0, T ; W^{1, p}(\Omega)\right)$ and $V^{*}$ be the dual space of $V$. The duality pairing between $V$ and $V^{*}$ will be denoted by $\langle\langle\cdot, \cdot\rangle\rangle_{V}$. The norm in $V$ will be denoted by $\|\cdot\|_{V}$, which is defined by

$$
\|u\|_{V}=\left(\int_{0}^{T}\|u(t)\|_{W^{1, p}(\Omega)}^{p} d t\right)^{\frac{1}{p}}, \quad \forall u(x, t) \in V
$$

Let $W=L^{q}\left(0, T ; W^{1, p}(\Omega)\right)$ and $W^{*}$ be the dual space of $W$. The norm in $W$ will be denoted by $\|\cdot\|_{W}$, which is defined by

$$
\|v\|_{W}=\left(\int_{0}^{T}\|v(t)\|_{W^{1, p}(\Omega)}^{q} d t\right)^{\frac{1}{q}}, \quad \forall v(x, t) \in W .
$$

In the integro-differential equation (1.11), $\Omega$ is a bounded conical domain of a Euclidean space $\mathbb{R}^{N}$ where $N \geq 1, \Gamma$ is the boundary of $\Omega$ with $\Gamma \in C^{1}[5], \vartheta$ denotes the exterior normal derivative to $\Gamma$. Here, $|\cdot|$ and $\langle\cdot, \cdot\rangle$ denote the Euclidean norm and the inner-product in $\mathbb{R}^{N}$, respectively. Also, $0 \leq C(x, t) \in L^{p}\left(0, T ; W^{1, p}(\Omega)\right), f(x, t) \in V^{*}$ is a given function, $T$ and $a$ are positive constants, and $\varepsilon$ is a nonnegative constant. Moreover, $\beta_{x}$ is the subdifferential of $\varphi_{x}$, where $\varphi_{x}=\varphi(x, \cdot): \mathbb{R} \rightarrow \mathbb{R}$ for $x \in \Gamma$, and $\varphi: \Gamma \times \mathbb{R} \rightarrow \mathbb{R}$ is a given function.

To tackle (1.11), we need the following assumptions which can be found in [5, 14].

Assumption 1 Green's formula is available.

Assumption 2 For each $x \in \Gamma, \varphi_{x}=\varphi(x, \cdot): \mathbb{R} \rightarrow \mathbb{R}$ is a proper, convex, and lowersemicontinuous function and $\varphi_{x}(0)=0$.

Assumption $30 \in \beta_{x}(0)$ and for each $t \in \mathbb{R}$, the function $x \in \Gamma \rightarrow\left(I+\lambda \beta_{x}\right)^{-1}(t) \in \mathbb{R}$ is measurable for $\lambda>0$.

We shall present a series of lemmas before we prove the main result.

Lemma 3.1 Define the function $\Phi: V \rightarrow \mathbb{R}$ by

$$
\Phi(u)=\int_{0}^{T} \int_{\Gamma} \varphi_{x}\left(\left.u\right|_{\Gamma}(x, t)\right) d \Gamma(x) d t, \quad \forall u \in V .
$$

Then $\Phi$ is a proper, convex, and lower-semicontinuous mapping on $V$. Therefore, $\partial \Phi: V \rightarrow$ $V^{\prime \prime}$, the subdifferential of $\Phi$, is maximal monotone.

Proof The proof of this lemma is analogous to that of Lemma 3.1 in [1]. We give the outline of the proof as follows.

Note that for each $s \in \mathbb{R}$, the function $x \in \Gamma \rightarrow \beta_{x}^{0}(s) \in \mathbb{R}$ is measurable, where $\beta_{x}^{0}(s)$ denotes the minimal section of $\beta_{x}$. Since for all $s_{1}, s_{2} \in \mathbb{R}$ we have

$$
\left\{x \in \Gamma: \varphi_{x}\left(s_{1}\right)>s_{2}\right\}=\bigcup_{n}\left\{x \in \Gamma: \sum_{i=1}^{n} \frac{s_{1}}{n} \beta_{x}^{0}\left(\frac{i s_{1}}{n}\right)>s_{2}\right\},
$$

it implies that for $u \in V$, the function $\varphi_{x}\left(\left.u\right|_{\Gamma}(x, t)\right)$ is measurable on $\Gamma$. Then from the property of $\varphi_{x}$, we know that $\Phi$ is proper and convex on $V$. 
To see that $\Phi$ is lower-semicontinuous on $V$, let $u_{n} \rightarrow u$ in $V$. We may assume that there exists a subsequence of $u_{n}$, for simplicity, we still denote it by $u_{n}$, such that $\left.u_{n}\right|_{\Gamma}(x, t) \rightarrow$ $\left.u\right|_{\Gamma}(x, t)$ for $x \in \Gamma$ and $t \in(0, T)$ a.e. This yields

$$
\varphi_{x}\left(\left.u\right|_{\Gamma}(x, t)\right) \leq \liminf _{n \rightarrow \infty} \varphi_{x}\left(\left.u_{n}\right|_{\Gamma}(x, t)\right)
$$

for all $x \in \Gamma$ and each $t \in(0, T)$ a.e. since $\varphi_{x}$ is lower-semicontinuous for each $x \in \Gamma$. It then follows from Fatou's lemma that for each $t \in(0, T)$,

$$
\begin{aligned}
\int_{\Gamma} \varphi_{x}\left(\left.u\right|_{\Gamma}(x, t)\right) d \Gamma(x) & \leq \int_{\Gamma} \liminf _{n \rightarrow \infty} \varphi_{x}\left(\left.u_{n}\right|_{\Gamma}(x, t)\right) d \Gamma(x) \\
& \leq \liminf _{n \rightarrow \infty} \int_{\Gamma} \varphi_{x}\left(\left.u_{n}\right|_{\Gamma}(x, t)\right) d \Gamma(x) .
\end{aligned}
$$

So, $\Phi(u) \leq \liminf _{n \rightarrow \infty} \Phi\left(u_{n}\right)$ whenever $u_{n} \rightarrow u$ in $V$. This completes the proof.

Lemma 3.2 Define $S: D(S)=\left\{u \in V: \frac{\partial u}{\partial t} \in V^{*}, u(x, 0)=u(x, T)\right\} \rightarrow V^{*}$ by

$$
S u=\frac{\partial u}{\partial t}+a \frac{\partial}{\partial t} \int_{\Omega} u d x .
$$

Then $S$ is a linear maximal monotone operator possessing a dense domain in $V$.

Proof It is obvious that $S$ is closed and linear.

For $u(x, t), w(x, t) \in D(S)$, integrating by parts gives

$$
\begin{aligned}
& \langle\langle w, S u\rangle\rangle_{V}+\left\langle\left\langle u, \frac{\partial w}{\partial t}+a \frac{\partial}{\partial t} \int_{\Omega} w d x\right\rangle\right\rangle_{V} \\
& =\int_{0}^{T} \int_{\Omega} \frac{\partial u}{\partial t} w(x, t) d x d t+a \int_{0}^{T} \int_{\Omega}\left(\frac{\partial}{\partial t} \int_{\Omega} u d x\right) w(x, t) d x d t \\
& \quad+\int_{0}^{T} \int_{\Omega} u(x, t) \frac{\partial w}{\partial t} d x d t+a \int_{0}^{T} \int_{\Omega}\left(\frac{\partial}{\partial t} \int_{\Omega} w d x\right) u(x, t) d x d t \\
& =\int_{\Omega} u(x, T) w(x, T) d x-\int_{\Omega} u(x, 0) w(x, 0) d x \\
& \quad+a \int_{\Omega} u(x, T) d x \int_{\Omega} w(x, T) d x-a \int_{\Omega} u(x, 0) d x \int_{\Omega} w(x, 0) d x=0 .
\end{aligned}
$$

Then $S^{*} w=-\frac{\partial w}{\partial t}-a \frac{\partial}{\partial t} \int_{\Omega} w d x$, where $D\left(S^{*}\right)=\left\{w \in V: \frac{\partial w}{\partial t} \in V^{*}, w(x, 0)=w(x, T)\right\}$.

For $u(x, t) \in D(S)$, we find

$$
\begin{aligned}
\int_{0}^{T} \int_{\Omega} \frac{\partial u}{\partial t} u(x, t) d x d t & =\int_{\Omega}|u(x, T)|^{2} d x-\int_{\Omega}|u(x, 0)|^{2} d x-\int_{0}^{T} \int_{\Omega} \frac{\partial u}{\partial t} u(x, t) d x d t \\
& =-\int_{0}^{T} \int_{\Omega} \frac{\partial u}{\partial t} u(x, t) d x d t
\end{aligned}
$$

which implies that

$$
\int_{0}^{T} \int_{\Omega} \frac{\partial u}{\partial t} u(x, t) d x d t=0
$$


Similarly, for $u(x, t) \in D(S)$,

$$
\begin{aligned}
& a \int_{0}^{T} \int_{\Omega} u(x, t)\left(\frac{\partial}{\partial t} \int_{\Omega} u d x\right) d x d t \\
& \quad=a\left(\int_{\Omega} u(x, T) d x\right)^{2}-a\left(\int_{\Omega} u(x, 0) d x\right)^{2}-a \int_{0}^{T} \int_{\Omega} u(x, t)\left(\frac{\partial}{\partial t} \int_{\Omega} u d x\right) d x d t,
\end{aligned}
$$

which implies that

$$
a \int_{0}^{T} \int_{\Omega} u(x, t)\left(\frac{\partial}{\partial t} \int_{\Omega} u d x\right) d x d t=0
$$

Thus,

$$
\langle\langle u, S u\rangle\rangle_{V}=\int_{0}^{T} \int_{\Omega} \frac{\partial u}{\partial t} u(x, t) d x d t+a \int_{0}^{T} \int_{\Omega} u(x, t)\left(\frac{\partial}{\partial t} \int_{\Omega} u d x\right) d x d t=0 .
$$

In the same manner, we have $\left\langle\left\langle w, S^{*} w\right\rangle\right\rangle_{V}=0$ for $w \in D\left(S^{*}\right)$. Therefore, noting Lemma 2.5 the result follows.

In view of Lemmas 2.3 and 2.4 , we have the following result.

Lemma 3.3 $S+\partial \Phi: V \rightarrow V^{*}$ is maximal monotone.

Lemma 3.4 [14] Define the mapping $B_{p, q}: W^{1, p}(\Omega) \rightarrow\left(W^{1, p}(\Omega)\right)^{*}$ as follows:

$$
\left(\bar{v}, B_{p, q} \bar{u}\right)=\int_{\Omega}\left\langle\left(C(x, t)+|\nabla \bar{u}|^{2}\right)^{\frac{p-2}{2}} \nabla \bar{u}, \nabla \bar{v}\right\rangle d x+\varepsilon \int_{\Omega}|\bar{u}|^{q-2} \overline{u v} d x, \quad \forall \bar{u}, \bar{v} \in W^{1, p}(\Omega) .
$$

Then $B_{p, q}$ is maximal monotone.

Lemma $3.5[14]$ Let $X_{0}$ denote the closed subspace of all constant functions in $W^{1, p}(\Omega)$. Let $X$ be the quotient space $\frac{W^{1, p}(\Omega)}{X_{0}}$. For $\bar{u} \in W^{1, p}(\Omega)$, define the mapping $P: W^{1, p}(\Omega) \rightarrow X_{0}$ by

$$
P \bar{u}=\frac{1}{\operatorname{meas}(\Omega)} \int_{\Omega} \bar{u} d x
$$

Then, there is a constant $C>0$ such that for every $\bar{u} \in W^{1, p}(\Omega)$,

$$
\|\bar{u}-P \bar{u}\|_{L^{p}(\Omega)} \leq C\|\nabla \bar{u}\|_{\left(L^{p}(\Omega)\right)^{N}}
$$

Here meas $(\Omega)$ denotes the measure of $\Omega$.

Definition 3.1 Define $A: V \rightarrow V^{*}$ as follows:

$$
\langle\langle v, A u\rangle\rangle_{V}=\int_{0}^{T}\left(v, B_{p, q} u\right) d t-\int_{0}^{T} \int_{\Omega} f(x, t) v(x, t) d x d t, \quad \forall u, v \in V .
$$

Lemma 3.6 The mapping $A: V \rightarrow V^{*}$ is everywhere defined, bounded, monotone, and hemi-continuous. Therefore, Lemma 2.2 implies that it is also pseudo-monotone. 
Proof From Lemma 2.1, we know that $W^{1, p}(\Omega) \hookrightarrow C_{B}(\Omega)$ when $p>N$, and $W^{1, p}(\Omega) \hookrightarrow$ $L^{q}(\Omega)$ when $p=N$. If $p<N$, then $W^{1, p}(\Omega) \hookrightarrow L^{\frac{N p}{N-p}}(\Omega) \hookrightarrow L^{p}(\Omega) \hookrightarrow L^{q}(\Omega)$ since $1<q \leq p<$ $+\infty$. Thus, for all $\bar{w} \in W^{1, p}(\Omega),\|\bar{w}\|_{L^{q}(\Omega)} \leq k\|\bar{w}\|_{W^{1, p}(\Omega)}$, where $k>0$ is a constant. Therefore, for $u, v \in V$, we have

$$
\int_{0}^{T}\|u\|_{L^{q}(\Omega)}^{q} d t \leq \mathrm{const} \cdot \int_{0}^{T}\|u\|_{W^{1, p}(\Omega)}^{q} d t=\mathrm{const} \cdot\|u\|_{W}^{q}
$$

and

$$
\int_{0}^{T}\|v\|_{L^{q}(\Omega)}^{q} d t \leq \mathrm{const} \cdot \int_{0}^{T}\|v\|_{W^{1, p}(\Omega)}^{q} d t=\text { const } \cdot\|v\|_{W}^{q} .
$$

Moreover, since $1<q \leq p<+\infty$, then $L^{p}\left(0, T ; W^{1, p}(\Omega)\right) \hookrightarrow L^{q}\left(0, T ; W^{1, p}(\Omega)\right)$, which implies that $\|u\|_{W} \leq\|u\|_{V}$ and $\|v\|_{W} \leq\|v\|_{V}$ for $u, v \in V$.

If $p \geq 2$, then for $u, v \in V$, we have

$$
\begin{aligned}
\left|\langle\langle v, A u\rangle\rangle_{V}\right| & \\
\leq & \left.\left.\int_{0}^{T} \int_{\Omega}|C(x, t)+| \nabla u\right|^{2}\right|^{\frac{p-2}{2}}|\nabla u| \cdot|\nabla v| d x d t \\
& +\varepsilon \int_{0}^{T} \int_{\Omega}|u|^{q-1}|v| d x d t+\int_{0}^{T} \int_{\Omega}|f| \cdot|v| d x d t \\
\leq & \int_{0}^{T} \int_{\Omega}\left|2 \max \left(C(x, t),|\nabla u|^{2}\right)\right|^{\frac{p-2}{2}}|\nabla u| \cdot|\nabla v| d x d t \\
& +\operatorname{const} \cdot \varepsilon\|v\|_{W}\|u\|_{W}^{\frac{q}{q^{\prime}}}+\|f\|_{V^{*}}\|v\|_{V} \\
\leq & 2^{\frac{p-2}{2}} \int_{0}^{T} \int_{\Omega} C(x, t)^{\frac{p-2}{2}}|\nabla u| \cdot|\nabla v| d x d t+2^{\frac{p-2}{2}}\|u\|_{V}^{\frac{p}{p^{p}}}\|v\|_{V} \\
& +\operatorname{const} \cdot \varepsilon\|v\|_{W}\|u\|_{W}^{\frac{q}{q^{\prime}}}+\|f\|_{V^{*}}\|v\|_{V} \\
\leq & 2^{\frac{p-2}{2}}\left(\int_{0}^{T} \int_{\Omega} C(x, t)^{\frac{p-2}{2} p^{\prime}}|\nabla v|^{p^{\prime}} d x d t\right)^{\frac{1}{p^{\prime}}}\|u\|_{V}+2^{\frac{p-2}{2}}\|u\|_{V}^{\frac{p}{p^{\prime}}}\|v\|_{V} \\
& +\operatorname{const} \cdot \varepsilon\|v\|_{W}\|u\|_{W}^{\frac{q}{q^{\prime}}}+\|f\|_{V^{*}}\|v\|_{V} \\
\leq & 2^{\frac{p-2}{2}}\|C(x, t)\|_{V}^{p-2}\|u\|_{V}\|v\|_{V}+2^{\frac{p-2}{2}}\|u\|_{V}^{\frac{p}{p^{\prime}}}\|v\|_{V}+\text { const } \cdot \varepsilon\|v\|_{V}\|u\|_{V}^{\frac{q}{q^{\prime}}}+\|f\|_{V^{*}}\|v\|_{V},
\end{aligned}
$$

which implies that $A$ is everywhere defined and bounded.

If $1<p<2$, then for $u, v \in V$, we have

$$
\begin{aligned}
& \left|\langle\langle v, A u\rangle\rangle_{V}\right| \\
& \leq\left.\left.\int_{0}^{T} \int_{\Omega}|C(x, t)+| \nabla u\right|^{2}\right|^{\frac{p-2}{2}}|\nabla u| \cdot|\nabla v| d x d t \\
& \quad+\varepsilon \int_{0}^{T} \int_{\Omega}|u|^{q-1}|v| d x d t+\int_{0}^{T} \int_{\Omega}|f| \cdot|v| d x d t \\
& =\int_{0}^{T} \int_{\Omega} \frac{|\nabla u| \cdot|\nabla v|}{\left.\left.|C(x, t)+| \nabla u\right|^{2}\right|^{\frac{2-p}{2}}} d x d t+\text { const } \cdot \varepsilon\|v\|_{W}\|u\|_{W}^{\frac{q}{q^{\prime}}}+\|f\|_{V^{*}}\|v\|_{V}
\end{aligned}
$$




$$
\begin{aligned}
& \leq \int_{0}^{T} \int_{\Omega} \frac{|\nabla u| \cdot|\nabla v|}{|\nabla u|^{2-p}} d x d t+\text { const } \cdot \varepsilon\|v\|_{W}\|u\|_{W}^{\frac{q}{q^{\prime}}}+\|f\|_{V^{*}}\|v\|_{V} \\
& \leq\|u\|_{V}^{\frac{p}{p^{\prime}}}\|v\|_{V}+\text { const } \cdot \varepsilon\|v\|_{V}\|u\|_{V}^{\frac{q}{q^{\prime}}}+\|f\|_{V^{*}}\|v\|_{V},
\end{aligned}
$$

which also implies that $A$ is everywhere defined and bounded.

Since $B_{p, q}$ is monotone, we can easily see that for $u, v \in V$,

$$
\langle\langle u-v, A u-A v\rangle\rangle_{V}=\int_{0}^{T}\left(u-v, B_{p, q} u-B_{p, q} v\right) d t \geq 0
$$

which implies that $A$ is monotone.

To show that $A$ is hemi-continuous, it suffices to show that for any $u, v, w \in V$ and $k \in$ $[0,1],\langle\langle w, A(u+k v)-A u\rangle\rangle_{V} \rightarrow 0$, as $k \rightarrow 0$. Noting the fact that $B_{p, q}$ is hemi-continuous and using the Lebesgue's dominated convergence theorem, we have

$$
0 \leq \lim _{k \rightarrow 0}\left|\langle w, A(u+k v)-A u\rangle_{V}\right| \leq \int_{0}^{T} \lim _{k \rightarrow 0}\left|\left(w, B_{p, q}(u+k v)-B_{p, q} u\right)\right| d t=0 .
$$

Hence, $A$ is hemi-continuous.

This completes the proof.

Lemma 3.7 The mapping $A: V \rightarrow V^{*}$ satisfies that for $u \in D(S)$,

$$
\frac{\left\langle\left\langle u-u_{0}, A u\right\rangle\right\rangle_{V}}{\|u\|_{V}} \rightarrow+\infty
$$

as $\|u\|_{V} \rightarrow+\infty$ in $V$.

Proof First, we shall show that for $u \in V$,

$$
\|u\|_{V} \rightarrow+\infty
$$

is equivalent to

$$
\left\|u-\frac{1}{\operatorname{meas}(\Omega)} \int_{\Omega} u d x\right\|_{V} \rightarrow+\infty
$$

In fact, from Lemma 3.5, we know that for $u \in V$,

$$
\left\|u-\frac{1}{\operatorname{meas}(\Omega)} \int_{\Omega} u d x\right\|_{L^{p}(\Omega)} \leq C\|\nabla u\|_{\left(L^{p}(\Omega)\right)^{N}},
$$

where $C$ is a positive constant. Thus,

$$
\begin{aligned}
\| u & -\frac{1}{\operatorname{meas}(\Omega)} \int_{\Omega} u d x \|_{W^{1, p}(\Omega)}^{p} \\
& =\left\|u-\frac{1}{\operatorname{meas}(\Omega)} \int_{\Omega} u d x\right\|_{L^{p}(\Omega)}^{p}+\left\|\nabla\left(u-\frac{1}{\operatorname{meas}(\Omega)} \int_{\Omega} u d x\right)\right\|_{\left(L^{p}(\Omega)\right)^{N}}^{p} \\
& \leq\left(C^{p}+1\right)\|\nabla u\|_{\left(L^{p}(\Omega)\right)^{N}}^{p},
\end{aligned}
$$


which implies that

$$
\begin{aligned}
\left\|u-\frac{1}{\operatorname{meas}(\Omega)} \int_{\Omega} u d x\right\|_{V} & \leq\left[\left(C^{p}+1\right) \int_{0}^{T}\|\nabla u\|_{\left(L^{p}(\Omega)\right)^{N}}^{p} d t\right]^{\frac{1}{p}} \\
& \leq\left(C^{p}+1\right)^{\frac{1}{p}}\|u\|_{V} .
\end{aligned}
$$

On the other hand, we have

$$
\left\|u-\frac{1}{\operatorname{meas}(\Omega)} \int_{\Omega} u d x\right\|_{W^{1, p}(\Omega)} \geq\|u\|_{W^{1, p}(\Omega)}-\left\|\frac{1}{\operatorname{meas}(\Omega)} \int_{\Omega} u d x\right\|_{W^{1, p}(\Omega)},
$$

which implies that

$$
\|u\|_{W^{1, p}(\Omega)} \leq\left\|u-\frac{1}{\operatorname{meas}(\Omega)} \int_{\Omega} u d x\right\|_{W^{1, p}(\Omega)}+\text { const. }
$$

Hence,

$$
\|u\|_{V} \leq\left\|u-\frac{1}{\operatorname{meas}(\Omega)} \int_{\Omega} u d x\right\|_{V}+\text { const. }
$$

In view of (3.2) and (3.3), we have shown that for $u \in V,\|u\|_{V} \rightarrow+\infty$ is equivalent to $\left\|u-\frac{1}{\operatorname{meas}(\Omega)} \int_{\Omega} u d x\right\|_{V} \rightarrow+\infty$.

Next, we shall show that $A$ satisfies (3.1). In fact, we have

$$
\begin{aligned}
& \frac{\left\langle\left\langle u-u_{0}, A u\right\rangle_{V}\right.}{\|u\|_{V}} \\
& =\frac{\int_{0}^{T} \int_{\Omega}\left\langle\left(C(x, t)+|\nabla u|^{2}\right)^{\frac{p-2}{2}} \nabla u, \nabla u\right\rangle d x d t}{\|u\|_{V}} \\
& \quad+\varepsilon \frac{\int_{0}^{T} \int_{\Omega}|u|^{q} d x d t}{\|u\|_{V}}-\frac{\int_{0}^{T} \int_{\Omega} f(x, t)\left(u-u_{0}\right) d x d t}{\|u\|_{V}} \\
& \quad-\frac{\int_{0}^{T} \int_{\Omega}\left\langle\left(C(x, t)+|\nabla u|^{2}\right)^{\frac{p-2}{2}} \nabla u, \nabla u_{0}\right\rangle d x d t}{\|u\|_{V}}-\varepsilon \frac{\int_{0}^{T} \int_{\Omega}|u|^{q-2} u u_{0} d x d t}{\|u\|_{V}} .
\end{aligned}
$$

If $1<p<2$, then

$$
\begin{aligned}
\frac{\int_{0}^{T} \int_{\Omega}\left\langle\left(C(x, t)+|\nabla u|^{2}\right)^{\frac{p-2}{2}} \nabla u, \nabla u\right\rangle d x d t}{\|u\|_{V}}+\varepsilon \frac{\int_{0}^{T} \int_{\Omega}|u|^{q} d x d t}{\|u\|_{V}} \\
=\frac{1}{\|u\|_{V}}\left[\int_{0}^{T} \int_{\Omega}\left(C(x, t)+|\nabla u|^{2}\right)^{\frac{p}{2}} d x d t\right. \\
\left.\quad-\int_{0}^{T} \int_{\Omega} \frac{C(x, t)}{\left(C(x, t)+|\nabla u|^{2}\right)^{\frac{2-p}{2}}} d x d t+\varepsilon \int_{0}^{T} \int_{\Omega}|u|^{q} d x d t\right] \\
\geq \frac{1}{\|u\|_{V}}\left[\int_{0}^{T} \int_{\Omega}|\nabla u|^{p} d x d t+\varepsilon \int_{0}^{T} \int_{\Omega}|u|^{q} d x d t\right] \\
\quad-\frac{1}{\|u\|_{V}} \int_{0}^{T} \int_{\Omega} \frac{C(x, t)}{\left(C(x, t)+|\nabla u|^{2}\right)^{\frac{2-p}{2}}} d x d t
\end{aligned}
$$




$$
\begin{aligned}
\geq & \frac{1}{\|u\|_{V}}\left[\int_{0}^{T} \int_{\Omega}|\nabla u|^{p} d x d t+\varepsilon \int_{0}^{T} \int_{\Omega}|u|^{q} d x d t\right] \\
& -\frac{1}{\|u\|_{V}} \int_{0}^{T} \int_{\Omega} \frac{C(x, t)}{C(x, t)^{\frac{2-p}{2}}} d x d t \\
\geq & \frac{1}{\|u\|_{V}} \int_{0}^{T} \int_{\Omega}|\nabla u|^{p} d x d t-\frac{1}{\|u\|_{V}} \int_{0}^{T} \int_{\Omega} C(x, t)^{\frac{p}{2}} d x d t .
\end{aligned}
$$

From (3.2) and (3.3), we know that

$$
\int_{0}^{T} \int_{\Omega}|\nabla u|^{p} d x d t \geq \frac{1}{C^{p}+1}\left\|u-\frac{1}{\operatorname{meas}(\Omega)} \int_{\Omega} u d x\right\|_{V}^{p} \geq \frac{1}{C^{p}+1}\|u\|_{V}^{p}+\text { const. }
$$

Also,

$$
\int_{0}^{T} \int_{\Omega} C(x, t)^{\frac{p}{2}} d x d t \leq\|C(x, t)\|_{V}^{p}<+\infty .
$$

It follows from (3.5) that

$$
\frac{\int_{0}^{T} \int_{\Omega}\left\langle\left(C(x, t)+|\nabla u|^{2}\right)^{\frac{p-2}{2}} \nabla u, \nabla u\right\rangle d x d t}{\|u\|_{V}}+\varepsilon \frac{\int_{0}^{T} \int_{\Omega}|u|^{q} d x d t}{\|u\|_{V}} \rightarrow+\infty,
$$

as $\|u\|_{V} \rightarrow+\infty$.

Moreover, we have

$$
\begin{aligned}
& \mid \frac{\int_{0}^{T} \int_{\Omega}\left\langle\left(C(x, t)+|\nabla u|^{2}\right)^{\frac{p-2}{2}} \nabla u, \nabla u_{0}\right\rangle d x d t}{\|u\|_{V}} \\
& +\varepsilon \frac{\int_{0}^{T} \int_{\Omega}|u|^{q-2} u u_{0} d x d t}{\|u\|_{V}}+\frac{\int_{0}^{T} \int_{\Omega} f(x, t)\left(u-u_{0}\right) d x d t}{\|u\|_{V}} \mid \\
& \leq \frac{\int_{0}^{T} \int_{\Omega}\left(C(x, t)+|\nabla u|^{2}\right)^{\frac{p-2}{2}}|\nabla u| \cdot\left|\nabla u_{0}\right| d x d t}{\|u\|_{V}} \\
& +\varepsilon \frac{\int_{0}^{T} \int_{\Omega}|u|^{q-1}\left|u_{0}\right| d x d t}{\|u\|_{V}}+\frac{\int_{0}^{T} \int_{\Omega}|f| \cdot\left|u-u_{0}\right| d x d t}{\|u\|_{V}} \\
& \leq \frac{1}{\|u\|_{V}} \int_{0}^{T} \int_{\Omega} \frac{|\nabla u| \cdot\left|\nabla u_{0}\right|}{\left(C(x, t)+|\nabla u|^{2}\right)^{\frac{2-p}{2}}} d x d t \\
& +\frac{\varepsilon}{\|u\|_{V}} \int_{0}^{T} \int_{\Omega}|u|^{q-1}\left|u_{0}\right| d x d t+\frac{\|f\|_{V^{n}}\left\|u-u_{0}\right\|_{V}}{\|u\|_{V}} \\
& \leq \frac{1}{\|u\|_{V}} \int_{0}^{T} \int_{\Omega}|\nabla u|^{p-1}\left|\nabla u_{0}\right| d x d t+\text { const } \cdot \frac{\varepsilon\|u\|_{V}^{\frac{q}{q^{\prime}}}\left\|u_{0}\right\|_{V}}{\|u\|_{V}}+\frac{\|f\|_{V^{*}}\left\|u-u_{0}\right\|_{V}}{\|u\|_{V}} \\
& \leq \frac{1}{\|u\|_{V}}\left[\|u\|_{V}^{\frac{p}{p^{\prime}}}\left\|u_{0}\right\|_{V}+\text { const } \cdot \varepsilon\|u\|_{V}^{\frac{q}{q^{\prime}}}\left\|u_{0}\right\|_{V}+\|f\|_{V^{*}}\left\|u_{0}\right\|_{V}\right]+\|f\|_{V^{*}} \\
& \leq \text { const. }
\end{aligned}
$$

Therefore, it follows from (3.4), (3.5), and (3.6) that $A$ satisfies (3.1) when $1<p<2$. 
If $p \geq 2$, then

$$
\begin{aligned}
& \frac{\left\langle\left\langle u-u_{0}, A u\right\rangle_{V}\right.}{\|u\|_{V}} \\
& \geq \frac{\int_{0}^{T} \int_{\Omega}\left\langle\left(C(x, t)+|\nabla u|^{2}\right)^{\frac{p-2}{2}} \nabla u, \nabla u\right\rangle d x d t}{\|u\|_{V}} \\
& +\varepsilon \frac{\int_{0}^{T} \int_{\Omega}|u|^{q} d x d t}{\|u\|_{V}}-\frac{\int_{0}^{T} \int_{\Omega}|f| \cdot\left|u-u_{0}\right| d x d t}{\|u\|_{V}} \\
& -\frac{\int_{0}^{T} \int_{\Omega}\left(C(x, t)+|\nabla u|^{2}\right)^{\frac{p-2}{2}}|\nabla u| \cdot\left|\nabla u_{0}\right| d x d t}{\|u\|_{V}}-\varepsilon \frac{\int_{0}^{T} \int_{\Omega}|u|^{q-1}\left|u_{0}\right| d x d t}{\|u\|_{V}} \\
& \geq \frac{\int_{0}^{T} \int_{\Omega}\left\langle\left(C(x, t)+|\nabla u|^{2}\right)^{\frac{p-2}{2}} \nabla u, \nabla u\right\rangle d x d t}{\|u\|_{V}}+\varepsilon \frac{\int_{0}^{T} \int_{\Omega}|u|^{q} d x d t}{\|u\|_{V}}-\frac{\|f\|_{V^{n}}\left\|u-u_{0}\right\|_{V}}{\|u\|_{V}} \\
& -\frac{2^{\frac{p-2}{2}} \int_{0}^{T} \int_{\Omega} C(x, t)^{\frac{p-2}{2}}|\nabla u| \cdot\left|\nabla u_{0}\right| d x d t}{\|u\|_{V}}-\frac{2^{\frac{p-2}{2}} \int_{0}^{T} \int_{\Omega}|\nabla u|^{p-1}\left|\nabla u_{0}\right| d x d t}{\|u\|_{V}} \\
& -\varepsilon \frac{\int_{0}^{T} \int_{\Omega}|u|^{q-1}\left|u_{0}\right| d x d t}{\|u\|_{V}} \\
& \geq \frac{\int_{0}^{T} \int_{\Omega}|\nabla u|^{p} d x d t}{\|u\|_{V}}-\frac{2^{\frac{p-2}{2}}\left(\int_{0}^{T} \int_{\Omega}|\nabla u|^{p} d x d t\right)^{\frac{1}{p^{p}}}\left(\int_{0}^{T} \int_{\Omega}\left|\nabla u_{0}\right|^{p} d x d t\right)^{\frac{1}{p}}}{\|u\|_{V}} \\
& -\frac{\|f\|_{V^{*}}\left\|u-u_{0}\right\|_{V}}{\|u\|_{V}}+\varepsilon \frac{\int_{0}^{T} \int_{\Omega}|u|^{q} d x d t}{\|u\|_{V}}-\varepsilon \frac{\left\|u_{0}\right\|_{V}\left(\int_{0}^{T} \int_{\Omega}|u|^{q} d x d t\right)^{\frac{1}{q^{\prime}}}}{\|u\|_{V}} \\
& -2^{\frac{p-2}{2}}\|C(x, t)\|_{V}^{p-2}\left\|u_{0}\right\|_{V} \\
& \geq \frac{M\left(\left\|u-\frac{1}{|\Omega|} \int_{\Omega} u d x\right\|_{V}^{p}-\left\|u_{0}\right\|_{V}\left\|u-\frac{1}{|\Omega|} \int_{\Omega} u d x\right\|_{V}^{\frac{p}{p^{\prime}}}\right)}{\|u\|_{V}}-\frac{\|f\|_{V^{n}}\left\|u-u_{0}\right\|_{V}}{\|u\|_{V}} \\
& +\frac{\varepsilon\left(\int_{0}^{T} \int_{\Omega}|u|^{q} d x d t\right)^{\frac{1}{q^{\prime}}}\left[\left(\int_{0}^{T} \int_{\Omega}|u|^{q} d x d t\right)^{1-\frac{1}{q^{\prime}}}-\left\|u_{0}\right\|_{V}\right]}{\|u\|_{V}} \\
& -2^{\frac{p-2}{2}}\|C(x, t)\|_{V}^{p-2}\left\|u_{0}\right\|_{V},
\end{aligned}
$$

where $M$ is a positive constant. We can easily see that

$$
\frac{\left\|u-\frac{1}{|\Omega|} \int_{\Omega} u d x\right\|_{V}^{p}-\left\|u_{0}\right\|_{V}\left\|u-\frac{1}{|\Omega|} \int_{\Omega} u d x\right\|_{V}^{\frac{p}{p^{\prime}}}}{\|u\|_{V}} \rightarrow+\infty,
$$

as $\|u\|_{V} \rightarrow+\infty$. Moreover, if $\int_{0}^{T} \int_{\Omega}|u|^{q} d x d t<+\infty$, then

$$
\frac{\varepsilon\left(\int_{0}^{T} \int_{\Omega}|u|^{q} d x d t\right)^{\frac{1}{q^{\prime}}}\left[\left(\int_{0}^{T} \int_{\Omega}|u|^{q} d x d t\right)^{1-\frac{1}{q^{\prime}}}-\left\|u_{0}\right\|_{V}\right]}{\|u\|_{V}} \rightarrow 0,
$$

as $\|u\|_{V} \rightarrow+\infty$; while if $\int_{0}^{T} \int_{\Omega}|u|^{q} d x d t \rightarrow+\infty$,

$$
\frac{\varepsilon\left(\int_{0}^{T} \int_{\Omega}|u|^{q} d x d t\right)^{\frac{1}{q^{\prime}}}\left[\left(\int_{0}^{T} \int_{\Omega}|u|^{q} d x d t\right)^{1-\frac{1}{q^{\prime}}}-\left\|u_{0}\right\|_{V}\right]}{\|u\|_{V}}>0 .
$$


Hence, the right side of (3.7) tends to $+\infty$ as $\|u\|_{V} \rightarrow+\infty$, which implies that $A$ satisfies (3.1).

This completes the proof.

Lemma 3.8 If $w(x, t) \in \partial \Phi(u)$, then $w(x, t)=\widetilde{w}(x, t) \in \partial \beta_{x}(u)$ a.e. on $\Gamma \times(0, T)$.

Proof If $w(x, t) \in \partial \Phi(u)$, then from the definition of subdifferential, we have

$$
\begin{aligned}
\int_{0}^{T} \int_{\Gamma} \varphi_{x}\left(\left.u\right|_{\Gamma}(x, t)\right) d \Gamma(x) d t \leq & \int_{0}^{T} \int_{\Gamma} \varphi_{x}\left(\left.w\right|_{\Gamma}(x, t)\right) d \Gamma(x) d t \\
& +\int_{0}^{T} \int_{\Gamma} w(x, t)(u-w) d \Gamma(x) d t
\end{aligned}
$$

which implies that the result is true.

We are now ready to prove the main result.

Theorem 3.1 The integro-differential equation (1.11) has a unique solution in $V$ for $f(x, t) \in V^{*}$.

Proof First, we shall show the existence of a solution. Noting Lemmas 2.6, 3.6, 3.7 and 3.3, and by using Theorem 2.1, we know that there exists $u(x, t) \in D(S) \subset V$ such that

$$
0=S u+A u+\partial \Phi(u) .
$$

Then we have for all $w \in V$,

$$
\langle\langle u-w, S u\rangle\rangle_{V}+\langle\langle u-w, A u\rangle\rangle_{V}+\langle u-w, \partial \Phi(u)\rangle_{V}=0 .
$$

The definition of subdifferential implies that

$$
\left\langle\left\langle u-w, \frac{\partial u}{\partial t}\right\rangle\right\rangle_{V}+\left\langle\left\langle u-w, a \frac{\partial}{\partial t} \int_{\Omega} u d x\right\rangle\right\rangle_{V}+\langle\langle u-w, A u\rangle\rangle_{V}+\Phi(u)-\Phi(w) \leq 0 .
$$

From the definition of $S$, we have

$$
u(x, 0)=u(x, T) .
$$

Moreover,

$$
\begin{aligned}
\int_{0}^{T} & \int_{\Omega} \frac{\partial u}{\partial t}(u-w) d x d t+\int_{0}^{T} \int_{\Omega}\left(a \frac{\partial}{\partial t} \int_{\Omega} u d x\right)(u-w) d x d t \\
+ & \int_{0}^{T} \int_{\Omega}\left\langle\left(C(x, t)+|\nabla u|^{2}\right)^{\frac{p-2}{2}} \nabla u, \nabla(u-w)\right\rangle d x d t \\
+ & \varepsilon \int_{0}^{T} \int_{\Omega}|u|^{q-2} u(u-w) d x d t \\
& -\int_{0}^{T} \int_{\Omega} f(x, t)(u-w) d x d t+\Phi(u)-\Phi(w) \leq 0 .
\end{aligned}
$$


Let $w=u \pm \psi$, where $\psi \in C_{0}^{\infty}(\Omega \times(0, T))$. Then we have

$$
\begin{aligned}
& \int_{0}^{T} \int_{\Omega} \frac{\partial u}{\partial t} \psi d x d t+\int_{0}^{T} \int_{\Omega}\left(a \frac{\partial}{\partial t} \int_{\Omega} u d x\right) \psi d x d t \\
& +\int_{0}^{T} \int_{\Omega}\left\langle\left(C(x, t)+|\nabla u|^{2}\right)^{\frac{p-2}{2}} \nabla u, \nabla \psi\right\rangle d x d t \\
& \quad+\varepsilon \int_{0}^{T} \int_{\Omega}|u|^{q-2} u \psi d x d t=\int_{0}^{T} \int_{\Omega} f(x, t) \psi d x d t .
\end{aligned}
$$

From the properties of a generalized function, we get

$$
\begin{aligned}
& \frac{\partial u}{\partial t}+a \frac{\partial}{\partial t} \int_{\Omega} u d x-\operatorname{div}\left[\left(C(x, t)+|\nabla u|^{2}\right)^{\frac{p-2}{2}} \nabla u\right]+\varepsilon|u|^{q-2} u \\
& \quad=f(x, t), \quad \text { a.e. in } \Omega \times(0, T) .
\end{aligned}
$$

Noting (3.10) again, by using Green's formula, we have

$$
\begin{aligned}
\int_{0}^{T} \int_{\Omega} & \frac{\partial u}{\partial t}(w-u) d x d t+\int_{0}^{T} \int_{\Omega}\left(a \frac{\partial}{\partial t} \int_{\Omega} u d x\right)(w-u) d x d t \\
& -\int_{0}^{T} \int_{\Omega} \operatorname{div}\left[\left(C(x, t)+|\nabla u|^{2}\right)^{\frac{p-2}{2}} \nabla u\right](w-u) d x d t \\
& +\left.\int_{0}^{T} \int_{\Gamma}\left\langle\vartheta,\left(C(x, t)+|\nabla u|^{2}\right)^{\frac{p-2}{2}} \nabla u\right\rangle(w-u)\right|_{\Gamma} d \Gamma(x) d t \\
& +\varepsilon \int_{0}^{T} \int_{\Omega}|u|^{q-2} u(w-u) d x d t+\Phi(w)-\Phi(u) \\
\geq & \int_{0}^{T} \int_{\Omega} f(x, t)(w-u) d x d t .
\end{aligned}
$$

Then using (3.10), we obtain

$$
\Phi(w)-\Phi(u) \geq-\left.\int_{0}^{T} \int_{\Gamma}\left\langle\vartheta,\left(C(x, t)+|\nabla u|^{2}\right)^{\frac{p-2}{2}} \nabla u\right\rangle(w-u)\right|_{\Gamma} d \Gamma(x) d t
$$

Thus, $-\left\langle\vartheta,\left(C(x, t)+|\nabla u|^{2}\right)^{\frac{p-2}{2}} \nabla u\right\rangle \in \partial \Phi(u)$.

In view of Lemma 3.8, we have $-\left\langle\vartheta,\left(C(x, t)+|\nabla u|^{2}\right)^{\frac{p-2}{2}} \nabla u\right\rangle \in \beta_{x}(u)$ a.e. on $\Gamma \times(0, T)$. Combining it with (3.8) and (3.11), we know that (1.11) has a solution in $V$.

Next, we shall prove the uniqueness of the solution. Let $u(x, t)$ and $v(x, t)$ be two solutions of (1.11). By (3.8), we have

$$
\langle u-v,(A+\partial \Phi) u-(A+\partial \Phi) v\rangle_{V}=-\langle\langle u-v, S u-S v\rangle\rangle_{V} \leq 0
$$

since $S$ is monotone. But $A+\partial \Phi$ is monotone too, so $\langle\langle u-v, S u-S v\rangle\rangle_{V}=0$, which implies that $u(x, t)=v(x, t)$.

The proof is complete. 


\section{Competing interests}

The authors declare that they have no competing interests.

\section{Authors' contributions}

All authors approve the final manuscript.

\section{Author details}

${ }^{1}$ School of Mathematics and Statistics, Hebei University of Economics and Business, Shijiazhuang, 050061, China.

${ }^{2}$ Department of Mathematics, Texas A\&M University — Kingsville, Kingsville, TX 78363, USA. ${ }^{3}$ Department of Mathematics, Faculty of Science, King Abdulaziz University, Jeddah, 21589, Saudi Arabia. ${ }^{4}$ School of Electrical and Electronic Engineering, Nanyang Technological University, 50 Nanyang Avenue, Singapore, 639798, Singapore.

\section{Acknowledgements}

Li Wei is supported by the National Natural Science Foundation of China (No. 11071053), the Natural Science Foundation of Hebei Province (No. A2010001482) and the Project of Science and Research of Hebei Education Department (the second round in 2010).

\section{Received: 13 June 2012 Accepted: 24 October 2012 Published: 13 November 2012}

\section{References}

1. Calvert, BD, Gupta, CP: Nonlinear elliptic boundary value problems in $L^{p}$-spaces and sums of ranges of accretive operators. Nonlinear Anal. 2, 1-26 (1978)

2. Gupta, CP, Hess, P: Existence theorems for nonlinear noncoercive operator equations and nonlinear elliptic boundary value problems. J. Differ. Equ. 22, 305-313 (1976)

3. Wei, L, He, Z: The applications of sums of ranges of accretive operators to nonlinear equations involving the p-Laplacian operator. Nonlinear Anal. 24,185-193 (1995)

4. Wei, L: The existence of solution of nonlinear elliptic boundary value problem. Math. Pract. Theory 31, 360-364 (2001) (in Chinese)

5. Wei, L, He, Z: The applications of theories of accretive operators to nonlinear elliptic boundary value problems in $L^{P}$-spaces. Nonlinear Anal. 46, 199-211 (2001)

6. Wei, L: The existence of a solution of nonlinear elliptic boundary value problems involving the $p$-Laplacian operator. Acta Anal. Funct. Appl. 4, 46-54 (2002) (in Chinese)

7. Wei, L: Study of the existence of the solution of nonlinear elliptic boundary value problems. Math. Pract. Theory 34 , 123-130 (2004) (in Chinese)

8. Wei, L, Zhou, H: The existence of solutions of nonlinear boundary value problem involving the $p$-Laplacian operator in $L^{5}$-spaces. J. Syst. Sci. Complex. 18, 511-521 (2005)

9. Wei, L, Zhou, H: Research on the existence of solution of equation involving the $p$-Laplacian operator. Appl. Math. J. Chin. Univ. Ser. B 21(2), 191-202 (2006)

10. Tolksdorf, P: On the Dirichlet problem for quasilinear equations in domains with conical boundary points. Commun Partial Differ. Equ. 8(7), 773-817 (1983)

11. Wei, L, Hou, W: Study of the existence of the solution of nonlinear elliptic boundary value problems. J. Hebei Norm. Univ. 28(6), 541-544 (2004) (in Chinese)

12. Wei, L, Zhou, H: Study of the existence of the solution of nonlinear elliptic boundary value problems. J. Math. Res Expo. 26(2), 334-340 (2006) (in Chinese)

13. Wei, L: The existence of solutions of nonlinear boundary value problems involving the generalized $p$-Laplacian operator in a family of spaces. Acta Anal. Funct. Appl. 7(4), 354-359 (2005) (in Chinese)

14. Wei, L, Agarwal, RP: Existence of solutions to nonlinear Neumann boundary value problems with generalized p-Laplacian operator. Comput. Math. Appl. 56(2), 530-541 (2008)

15. Wei, L, Agarwal, RP, Wong, PJY: Existence of solutions to nonlinear parabolic boundary value problems with generalized p-Laplacian operator. Adv. Math. Sci. Appl. 20(2), 423-445 (2010)

16. Zeilder, E: Nonlinear Functional Analysis and Its Applications. Springer, New York (1990)

17. Barbu, V: Nonlinear Semigroups and Differential Equations in Banach Spaces. Noordhoff, Leyden (1976)

18. Pascali, D, Sburlan, S: Nonlinear Mappings of Monotone Type. Sijthoff and Noordhoff, The Netherlands (1978)

19. Adams, RA: The Sobolev Space. People's Education Press, China (1981) (Version of Chinese Translation)

20. Lions, JL: Quelques Methods de Resolution des Problems aux Limites Nonlineaires. Dunod Gauthier-Villars, Paris (1969)

doi:10.1186/1687-2770-2012-131

Cite this article as: Wei et al.: Study on integro-differential equation with generalized $p$-Laplacian operator. Boundary Value Problems 2012 2012:131. 